\title{
Optical system design for micro star sensor
}

\author{
Tuo Zheng, Haiyang Quan \\ Beijing Microelectronics Technology Institute, Beijing 100076, China; \\ 2217404576@qq.com
}

Keywords: Optical system, star sensor,Image processing

\begin{abstract}
Star sensor is an important part of the attitude control of a space vehicle. The advantages and disadvantages of the optical system determine the accuracy of the whole star sensor system. In order to adapt to the development of China's space needs, a star sensor optical system was designed by a domestic CMOS sensor, domestic FPGA and domestic CPU. Its focal length $\mathrm{F} / \#=1: 1.73$, $\mathrm{f}=43.56 \mathrm{~mm}$, working wavelength range: $400 \mathrm{~nm} \sim 780 \mathrm{~nm}$, field of view angle: 20 degrees $\times 20$ degrees. Meet the requirements of the optical system of star sensor, and also meet the relevant technical indicators of the star sensor national standard 30111-2013 GB/T.
\end{abstract}

\section{Introduction}

Along with the continuous advancement of technology, as the outcome of optical remote sensing technology, star sensor plays a significant role in controlling posture. When spacecraft adjust its posture, star sensor needs to measure its posture accurately. It is mainly used in observing star in space, which tests weak signal. It can measure the posture by observing the position of star. Optical system is used to identify guide star in field of view which can present image on the sensor chip. And then, the electrical signal is processed by a series of image processing and compared with star database, so as to obtain the flight attitude [1-2]. Star sensor in the future direction of development will be small type. At the same time, as an important part of the space flight, the accuracy of star sensor is very important.

\section{Determination of parameters of optical system}

The parameters of the optical system of the star sensor mainly include the focal length, the working wavelength, the field of view, the aperture, and so on. Optical system parameters include the stars image parameters of concentrated energy, signal to noise ratio and integral time, minimum angle of view, limiting magnitude, and capture probability of 4 stars above navigation star and so on.

\subsection{Determination of field of view}

The angle of view is the parameter of determining the maximum range of the optical lens. Under the same conditions, the larger the field of view, the more stars can be observed. But too many stars will interfere with the subsequent calculation, so choosing the appropriate field of view is the first step in building an optical system.

The star sensor can detect the light of star sensitivity in dark, the size of pixel is $15 \mu \mathrm{m}$, resolution is $1024 \times 1024$, operating wavelength range is $400 \mathrm{~nm} \sim 780 \mathrm{~nm}$. The probability of capturing more than 4 navigation star at any posture is $99 \%$. According to this data, we can acquire the number of each magnitude of stars in field of view by programming and processing the star catalog. Through the statistics, in the limiting magnitude for $5,5.5,6$, 6.5, field of view were 23 degrees $\times 23$ degrees, 16 degrees $\times 16$ degrees, 12degrees $\times 12$ degrees and 9 degrees $\times 9$ degrees, the probability of appearing 4 guide stars or more were $99.84 \%$, 99.76\%, 99.75\% and 99.75\%. By further statistic, when chose the magnitude 5.5, select the viewing angle of 20 degrees $\times 20$ degrees can ensure 4 guide stars or more $t$ in any field[3]. Therefore, the angle of view is selected 20 degrees $\times 20$ degrees. 
Table 1 Number of stars at 20 degrees x 20 degrees

\begin{tabular}{|c|c|c|c|c|}
\hline star magnitude & 4.5 & 5.0 & 5.5 & 6.0 \\
\hline Less than 3 & $5.31 \%$ & $0.32 \%$ & 0 & 0 \\
\hline 3 & $5.67 \%$ & $1.06 \%$ & 0 & 0 \\
\hline 4 or more & $89.02 \%$ & $98.63 \%$ & $100 \%$ & $100 \%$ \\
\hline 8 or more & $47.62 \%$ & $89.44 \%$ & $99.9 \%$ & $100 \%$ \\
\hline 12 or more & $18.16 \%$ & $61.52 \%$ & $98.19 \%$ & $99.98 \%$ \\
\hline 16 or more & $8.83 \%$ & $33.64 \%$ & $92.1 \%$ & $99.88 \%$ \\
\hline 20 or more & $4.52 \%$ & $20.16 \%$ & $74.23 \%$ & $99.62 \%$ \\
\hline
\end{tabular}

\subsection{Focal length determination}

The focal length is the distance which is between imaging plane and the lens. The focal length can be changed correspondingly as a result of the selected object differently. In space, the position and distance of the stars are constant, thus the focal length of the star sensor is different from the ordinary camera. According to the formula of optical system field of view and the focal length [4-5]: $\tan \frac{W}{2}=\frac{\sqrt{a^{2}+b^{2}}}{2 f}$.

(A,B as the sensor side, $\mathrm{W}$ is the size of field of view, $\mathrm{f}$ is the focal length.)

It can be known that $\mathrm{f}=43.56 \mathrm{~mm}$.

\subsection{Determination of dispersion speckle size}

Taking example for 20 degrees $\times 20$ degrees field of view angle, by using $1024 \times 1024$ pixels, a single pixel can only reach 20/1024 $=0.0195$ degree $=70 "$. In order to improve accuracy of measurement of pixel, it needs to defocus the image which received by the sensor, and disperse the image point expanding to a number of pixels. Calculating and confirming the star position by using a plurality of pixel signal energy, thus the precision can reach to sub-pixel level. This is the extraction method of sub-pixel interpolation point. At present, two approaches can be used, which are $2 \times 2$ pixels or $3 \times 3$ pixels .Larger speckle could improve the positioning accuracy, but it will affect the calculation speed. In this paper, the size of $2 \times 2$ pixels is used as the speckle size.

\subsection{Determination of relative aperture}

The relative aperture is mainly composed of the limiting magnitude, noise, speckle size, energy concentration, integral time and other parameters to determine.

The maximum capacity which optical system obtain point light source in unit time is only related to the area of the lens aperture. When the sensor and the video processing circuit be determined, the limiting magnitude of the star which detected by the sensor depends on the aperture D of optical system. The larger aperture is, the more sensitive optical system has. However, with the aperture size getting larger, the aberration of the optical system will increase. Therefore, the size of the aperture should be limited, which maintains a certain relationship between the focal length and apertures. This relationship is usually represented by the relative aperture $F / \#=f / D$. In Chinese national standard, 30111-2013 GB/T, the relative aperture is defined as the ratio between the entrance pupil diameter and the focal length, that is, $\mathrm{D} / \mathrm{f}$ '. Required values for: between 1/0.8 1/6. $\mathrm{F} / \#$ is calculated by the following formula[6]:. is the signal to noise ratio which value 5 ; is dark current noise; is the sum of the rest of the noise; is optical system transmittance which value 0.75 ; for energy concentration which is $90 \%$; $t$ is the time integrated exposure which value $100 \mathrm{~ms}$; a represents pixel size which value is $15 \mu \mathrm{m} ; \mathrm{K}$ equivalent to a detector of the optical power; $\mathrm{H}$ means illumination which is emitted from star to optical system. QE is quantum efficiency; FF is the area occupied ratio; $\mathrm{d}$ is the speckle size, $\mathrm{h}$ is the Planck constant, $\mathrm{v}$ is the frequency of the incident light wavelength. The final results for $D=25.2 \mathrm{~mm}$. And $\mathrm{F} / \#=25.2 / 43.56=1: 1.73$. Comply with national standards 30111-2013 GB/. 


\section{Optical system designs}

After optimization of the optical system structure and optimization design, the optical system is shown in Figure 1 below. The detecting sensor can be directly packaged with the lens, replacing the conventional plate glass window, which is beneficial to the miniaturization of the lens, and reduces the stray light. The use of a transparent film can improve the transmission rate of the system to meet the requirements of the index. The optical system point list is shown in Figure 2, the visible dispersion pattern is basically circular, and basically falls within $2 \times 2$ pixels.

The diffraction energy concentration is shown in Figure 3.It can be seen to meet the normal distribution, and more than 95\% of the energy is concentrated in the radius of $15 \mu \mathrm{m}$.

The distortion curve is shown in Figure 4. The absolute value of the maximum distortion is located at 1.0 , and is equal to $0.2 \%$.

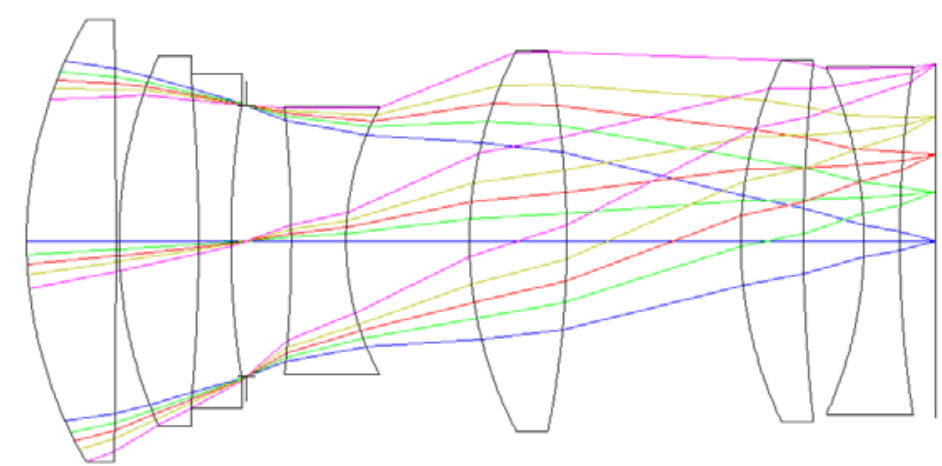

Fig. 1 optical system

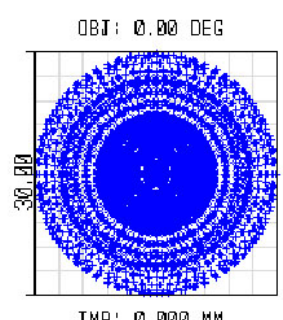

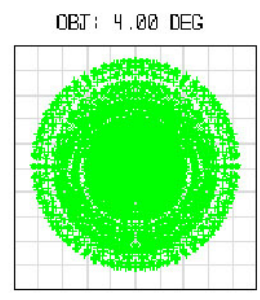

IMA: $3.035 \mathrm{MM}$
DET: $7.00 \mathrm{DEC}$

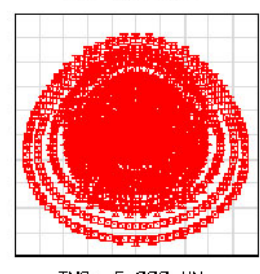

IMA: 5,333 HM
$\mathrm{OBJ}: 10,0 \mathrm{OB} \mathrm{CE}$

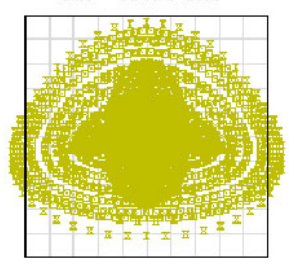

IMH: $7.668 \mathrm{MM}$
OEJ : 14.00 DEE

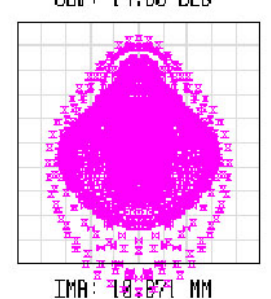

Fig. 2 Spot diagram 


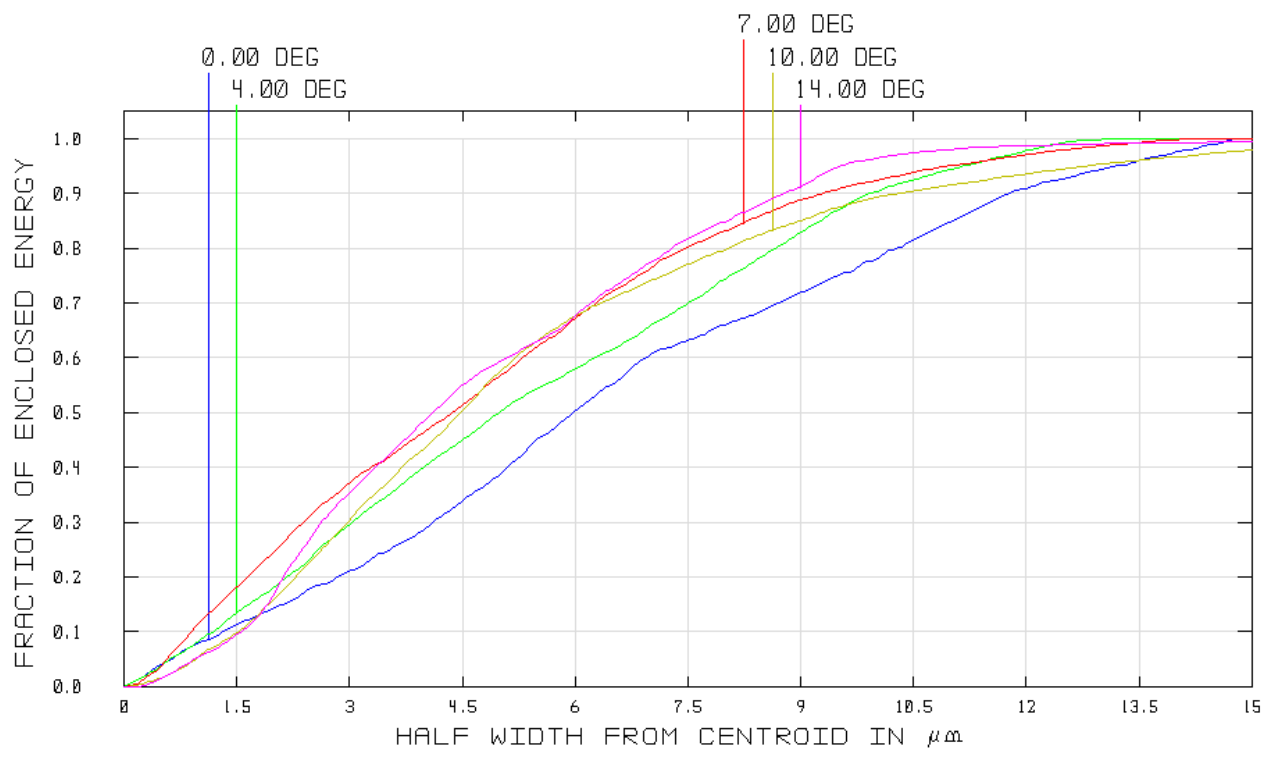

Fig. 3 Energy diffraction concentration DISTORTION

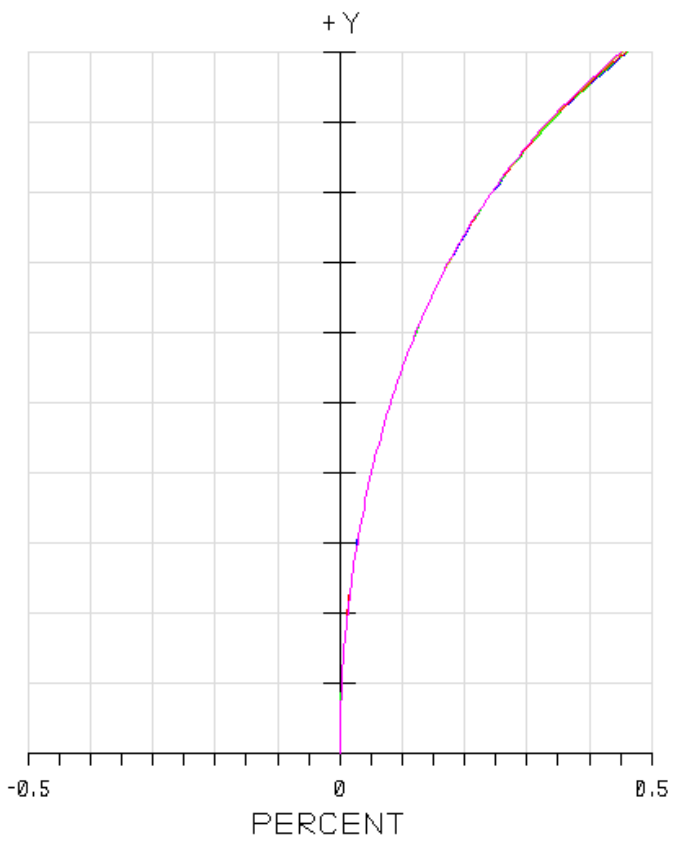

Fig. 4 Distortion curve diagram

\section{Conclusions}

A star sensor optical system based on domestic sensor chip is designed. In the process of localization and miniaturization, we must first understand the performance of the chip. From the angle of view of the field of view, the focal length and the relative aperture of the relative aperture are determined. According to the national standard 30111-2013 GB/T appropriate adjustment parameters to meet their requirements.

\section{Reference}

[1] Li Ping. Study on the improvement of measurement accuracy of star sensor [D]. Suzhou: Soochow University, 2006:8-9.

[2] Zheng Jiang. Research on the technology of light and small high dynamic star sensor [D]. 


\section{Shanghai:}

Shanghai Jiao Tong University, 2012:6-9.

[3] Wu Feng. Research on the key technologies of autonomous navigation star sensor [D]. Suzhou: Soochow University, 2012:3-4.

[4] zhengru, Guo Yu Zhang, Gao Yue, et. Based on LCOS splicing technology of dynamic star simulator, optical system design [J]. Instruments of, 2012, 33 (10): 2144-2150.

[5] Zhang Guoyu, Sun Xiangyang, Wang Dayi, et al.

Mechanical structure design of [J]. Chinese Journal of scientific instrument, 2011,32 (10): 2122-2125.

[6] Wu Feng. Research on the key technologies of autonomous navigation star sensor [D]. Suzhou: Soochow University, 2012:3-4. 